\title{
Unvail the Mysterious of the Single Spin Asymmetry
}

\author{
FENG YUAN \\ Nuclear Science Division, Lawrence Berkeley National Laboratory, Berkeley, CA 94720, USA \\ RIKEN/BNL Research Center, Building 510A, Brookhaven National Laboratory, Upton, NY \\ 11973
}

\begin{abstract}
Single transverse-spin asymmetry in high energy hadronic reaction has been greatly investigated from both experiment and theory sides in the last few years. In this talk, I will summarize some recent theoretical developments, which, in my opinion, help to unvail the mysterious of the single spin asymmetry.
\end{abstract}

Keywords: Single Spin Asymmetry; QCD Factorization.

PACS Nos.: 12.38.Bx, 13.88.+e, 12.39.St

\section{Introduction}

There have been strong experimental interests on transverse spin physics around the world, from the deep inelastic scattering experiments such as the HERMES collaboration at DESY, SMC at CERN, and Hall A and CLAS at JLab, the proton-proton collider experiment from RHIC at Brookhaven, and the very relevant $e^{+} e^{-}$annihilation experiment from BELLE at KEK. One of the major goals in transverse spin physics is to study the quark transversity distribution, the last unknown leading-twist quark distribution in nucleon. Besides the quark transversity distribution, the transverse spin physics also opens a new window to explore the partonic structure of nucleon, the so-called transverse momentum dependent (TMD) parton distributions. TMD parton distribution is an extension to the usual Feynman parton distributions. They allow us to study the three-dimension picture of partons inside the nucleon, and they are also closely related to the generalized parton distributions and the parton orbital angular momenta. Especially, the single transverse spin asymmetry (SSA) phenomena in high energy hadronic processes have attracted many theoretical and experimental investigations. The SSA is defined as the asymmetry when one of the hadrons' transverse spin is flipped, $A_{N} \sim\left(d \sigma\left(S_{\perp}\right)-d \sigma\left(-S_{\perp}\right)\right) /\left(d \sigma\left(S_{\perp}\right)-d \sigma\left(-S_{\perp}\right)\right)$. It has been a great theoretical challenge in the understanding of these phenomena.

It was the pioneer works by Efremov-Teryaev ${ }^{1}$ and Qiu-Sterman ${ }^{2}$, among others, to investigate this phenomena in the QCD framework beyond the naive parton picture, since the latter predicts very small single spin asymmetry in hard partonic scattering processes ${ }^{3}$. Recent theoretical developments have made great progress in the exploration of these physics. They have demonstrated a promise to unvail the 
mysterious for the single spin asymmetry. In this talk, I will try to summarize the current theory status. Of course, it is impossible to cover all the exciting achievements in recent years in this short presentation. Rather, I would like to focus on one important subject, i.e., the nontrivial QCD dynamics associated with transverse spin physics: the QCD factorization, the universality of the parton distributions and fragmentation functions, and their scale evolutions. These developments have laid solid theoretical foundation to apply QCD theory for the description of the single spin asymmetry phenomena. Fortunately, in this conference, there have been excellent talks on other developments in the transverse spin physics ${ }^{4}$, which compensate my omission of other important works.

\section{Universality and Factorization for the Transverse Momentum Dependent Functions}

Among those TMD parton distributions and fragmentation functions, two functions have been mostly discussed: the Sivers quark distribution ${ }^{5}$ and the Collins fragmentation function ${ }^{6}$. The Sivers quark distribution represents a distribution of unpolarized quarks in a transversely polarized nucleon, through a correlation between the quark's transverse momentum and the nucleon polarization vector. The Collins function represents a correlation between the transverse spin of the fragmenting quark and the transverse momentum of the hadron relative to the "jet axis" in the fragmentation process. Although they both belong to the so-called "naive-time-reversal-odd" functions, they do have different universality properties. For the quark Sivers function, because of the initial/final state interaction difference, they differ by signs for the SIDIS and Drell-Yan processes ${ }^{7,8,9,10}$. On the other hand, there have been several studies $11,12,13,14,15$ showing that the Collins function is universal between different processes, primarily in the SIDIS and $e^{+} e^{-}$ annihilation and recently in $p p$ collisions. In the following, I will take the example of the Collins contribution to the azimuthal asymmetric distribution of hadrons inside a high energy jet in the transversely polarized $p p$ collision to demonstrate this universality property ${ }^{14}$,

$$
p\left(P_{A}, S_{\perp}\right)+p\left(P_{B}\right) \rightarrow j e t\left(P_{J}\right)+X \rightarrow H\left(P_{h}\right)+X,
$$

where a transversely polarized proton with momentum $P_{A}$ scatters on another proton with momentum $P_{B}$, and produces a jet with momentum $P_{J}$. The three momenta of $P_{A}, P_{B}$ and $P_{J}$ form the so-called reaction plane. Inside the produced jet, the hadrons are distributed around the jet axis, where we define transverse momentum $P_{h T}$ relative to the jet axis. The correlation between $P_{h T}$ and the polarization vector $S_{\perp}$ introduces the Collins contribution to the single spin asymmetry in this process.

We need to generate a phase from the scattering amplitudes to have a nonvanishing SSA. If the phase comes from the vertex associated with the fragmenting quark and the final state hadron, or from the dressed quark propagator, it is 


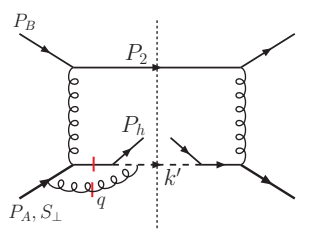

(a)

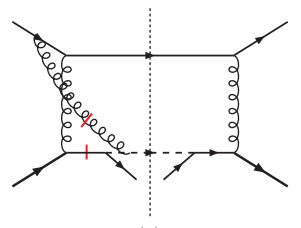

(c)

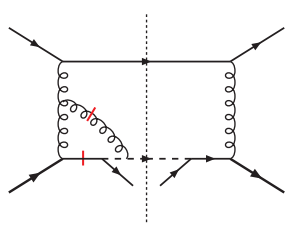

(b)

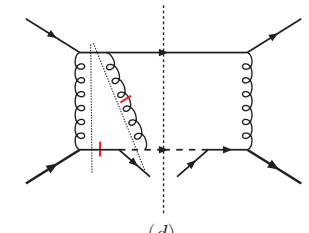

(d)

Fig. 1. Gluon exchange diagrams contributions to the Collins asymmetry in pp collisions. The short bars indicate the pole contributions to the phase needed for a non-vanishing SSA. The additional two cuts in (d) cancel out each other.

easy to argue the universality of the Collins function between this process and the SIDIS $/ e^{+} e^{-}$process, because they are the same. The main issue of the universality discussion concerns the extra gluon exchange contribution between the spectator of the fragmentation process and hard partonic part. In Fig. 2, we have shown all these interactions for a particular partonic channel $q q^{\prime} \rightarrow q q^{\prime}$ contribution, including the gluon attachments to the incident quarks (a,c), and final state balancing quark (d) and the internal gluon propagator (b). The contributing phases of the diagrams in Fig. 2 come from the cuts through the internal propagators in the partonic scattering amplitudes. In Fig. 2, we labeled these cut-poles by short bars in the diagrams. From the calculations, we will find that all these poles come from a cut through the exchanged gluon and the fragmenting quark in each diagram, and all other contributions either vanish or cancel out each other. For example, in Fig. 2(d), we show two additional cuts, which contribute however opposite to each other and cancel out completely. Therefore, by using the Ward identity at this particular order, the final results for all these diagrams will sum up together into a factorized form, where the cross section is written as the hard partonic cross section for $q\left(S_{\perp}\right) q^{\prime} \rightarrow q\left(s_{\perp}\right) q^{\prime}$ subprocess multiplied by a Collins fragmentation function. The exchanged gluon in Fig. 2 is now attaching to a gauge link from the fragmentation function definition. Similar calculations can be performed for the other two processes SIDIS and $e^{+} e^{-}$ annihilation, and the same Collins function will be observed. This argument can also be extended to two-gluon exchange diagrams ${ }^{14}$.

The key steps in the above derivation are the eikonal approximation and the Ward identity. The eikonal approximation is valid when we calculate the leading power contributions in the limit of $P_{h T} \ll P_{J}$. The Ward identity ensure that when we sum up the diagrams with all possible gluon attachments we shall get the eikonal propagator from the gauge link in the definition of the fragmentation function. The 
most important point to apply the Ward identity in the above analysis is that the eikonal propagator does not contribute to the phase needed to generate a nonzero SSA.

This observation is very different from the SSAs associated with the parton distributions, where the eikonal propagators from the gauge link in the parton distribution definition play very important role ${ }^{7,8,9,10}$. It is the pole of these eikonal propagators that contribute to the phase needed for a nonzero SSA associated with the naive-time-reversal-odd parton distributions, which also predicts a sign difference for the quark Sivers function between the SIDIS and Drell-Yan processes. More complicated results have been found for the SSAs in the hadronic dijet-correlation ${ }^{16,17}$, where a normal TMD factorization breaks down ${ }^{18}$. The reason is that the eikonal propagators from the initial and final state interactions in dijet-correlation process do contribute poles in the cross section ${ }^{17,18}$. Because of this, the Ward identity is not applicable, and the standard TMD factorization breaks down, although a modified factorization may be valid if we modify the definition of the TMD parton distributions to take into account all the initial and final state interaction effects ${ }^{16}$.

In particular, there is a sign change between the SSAs in SIDIS and Drell-Yan processes $^{7,8}$,

$$
\text { Sivers SSA }\left.\right|_{\mathrm{DY}}=- \text { Sivers SSA }\left.\right|_{\text {DIS }} \text {. }
$$

This nontrivial result of the opposite signs between the above two processes will still hold when gluon radiation contributions are taken into account, where the large transverse momentum Sivers function is generated from the twist-three quark-gluon correlation function ${ }^{19}$. It is of crucial to test this nontrivial QCD predictions by comparing the SSAs in these two processes. The Sivers single spin asymmetry in SIDIS process has been observed by the HERMES collaboration, and the planned Drell-Yan measurement at RHIC and other facility will test this prediction.

\section{Unifying the Two Mechanisms for SSA}

There are mainly two approaches to explore the single spin asymmetry phenomena in the QCD framework: the transverse momentum dependent approach discussed in the previous section and the collinear twist-three quark-gluon correlation approach pioneered by Efremov-Teryaev and Qiu-Sterman mentioned in the Introduction. Both approaches have been used to describe the experimental data on single spin asymmetry. One of the important developments in the last few years is that it has been shown that these two mechanisms are unified to describe the same physics ${ }^{19,20,21,22,23,24}$, in particular, in the semi-inclusive hadron production in Deep Inelastic Scattering (SIDIS) and Drell-Yan lepton pair production in hadronic collisions. For example, in SIDIS, at large $P_{h \perp} \sim Q$, the quark-gluon correlation approach applies. At small $P_{h \perp} \ll Q$, a factorization in terms of TMD parton distribution applies ${ }^{25}$, involving in case of the SSA the Sivers functions. If $P_{h \perp}$ is much larger than $\Lambda_{\mathrm{QCD}}$, the dependence of these functions on transverse momentum can be computed using QCD perturbation theory. At the same time, the 


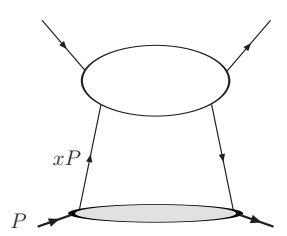

(a)

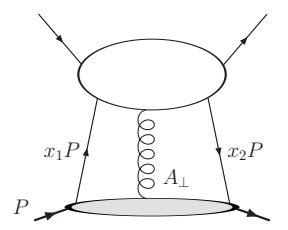

(c)

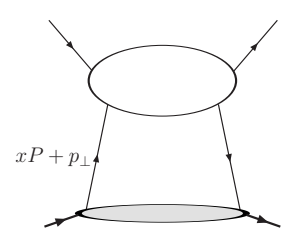

(b)

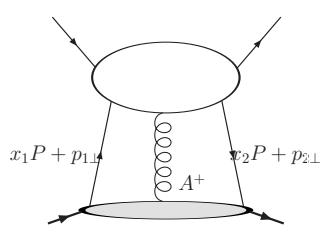

(d)

Fig. 2. Generic diagram interpretations for the twist expansions in the high energy scattering amplitudes up to twist-three level: (a) corresponds to a leading twist matrix element $\langle\bar{\psi} \psi\rangle$; (b)-(d) for twist-three contributions, (b) for $\left\langle\bar{\psi} \partial_{\perp} \psi\right\rangle$, (c) for $\left\langle\bar{\psi} A_{\perp} \psi\right\rangle$, and (d) for $\left\langle\bar{\psi} \partial_{\perp} A^{+} \psi\right\rangle$. Additional $A^{+}$gluon connection between hard partonic part and the non-perturbative nucleon structure part can be added to these diagrams. This is because they do not change the power counting in these diagrams. The contributions from these diagrams $(b-d)$ are not gauge invariant individually. However, they will combine into the gauge invariant results in terms of the correlation functions introduced in Sec.II.

result obtained within the twist-three formalism can also be extrapolated into the regime $\Lambda_{\mathrm{QCD}} \ll P_{h \perp} \ll Q$, and it has been demonstrated that the result of this extrapolation is identical to that obtained using the TMD approach.

The key step to demonstrate this consistency is the relation between the TMD quark distributions and the collinear leading twist and higher-twist distribution and fragmentation functions. In particular, the transverse momentum dependence can be calculated within the perturbative QCD and related to the collinear matrix elements as long as the $k_{\perp}$ is much larger than $\Lambda_{Q C D}$. In general, we will have the following expression for the quark distributions at large transverse momentum ${ }^{25}$,

$$
\left.q\left(x, k_{\perp}\right)\right|_{k_{\perp} \gg \Lambda_{\mathrm{QCD}}}=\frac{1}{\left(k_{\perp}^{2}\right)^{n}} \int \frac{d x^{\prime}}{x^{\prime}} f_{i}\left(x^{\prime}\right) \times \mathcal{H}_{q / i}\left(x ; x^{\prime}\right),
$$

where $q\left(x, k_{\perp}\right)$ represents the TMD quark distribution we are interested, $f_{i}$ represents the integrated quark distribution for the $k_{\perp}$-even TMDs, and higher twist quark-gluon correlation function for the $k_{\perp}$-odd TMDs. For the latter case, $x^{\prime}$ should be understood as two variables for the twist-three quark-gluon correlation functions as we discussed in the last section. The overall power behavior $1 /\left(k_{\perp}^{2}\right)^{n}$ can be analyzed by the power counting rule. The hard coefficient $\mathcal{H}_{q / i}\left(x ; x^{\prime}\right)$ is calculated from perturbative QCD.

To calculate the $k_{\perp}$-odd quark distribution, we make use of the twist expansion. In the twist expansion, a set of non-perturbative matrix elements of the hadron state will be analyzed according to the power counting of the associated contributions. 
At the twist-three order, from a generic power counting we have contributions from the following matrix elements,

$$
\left\langle\bar{\psi} \partial_{\perp} \psi\right\rangle, \quad\left\langle\bar{\psi} A_{\perp} \psi\right\rangle, \quad\left\langle\bar{\psi} \partial_{\perp} A^{+} \psi\right\rangle .
$$

We illustrate the typical diagrams for the associated contributions from the above matrix elements in Fig. 1. For comparison, we have also shown the diagram corresponding to the leading-twist contribution from the matrix element $\langle\bar{\psi} \psi\rangle$ in Fig. 1a. Figs. 1b-d represent the contributions up to twist-three quark-gluon correlation matrix elements. Fig. 1b corresponds to the contributions from the matrix element $\left\langle\bar{\psi} \partial_{\perp} \psi\right\rangle$, Fig. 1c from $\left\langle\bar{\psi} A_{\perp} \psi\right\rangle$, and Fig. 1d from $\left\langle\bar{\psi} \partial_{\perp} A^{+} \psi\right\rangle$. Because of additional gluon component in the matrix elements for Fig. $1 \mathrm{c}$ and $\mathrm{d}$, there will be gluon attachment from the nonperturbative part to the perturbative part as shown in these diagrams. To calculate the contributions from Fig. $1 \mathrm{~b}$ and d, we have to do collinear expansion of the partonic scattering amplitudes in terms of $p_{\perp}^{\alpha}$ and $k_{g \perp}^{\alpha}=p_{2 \perp}^{\alpha}-p_{1 \perp}^{\alpha}$, respectively. These expansions, combining with the quark field and gluon field, will lead to the contributions in terms of the matrix elements: $\left\langle\bar{\psi} \partial_{\perp} \psi\right\rangle$, and $\left\langle\bar{\psi} \partial_{\perp} A^{+} \psi\right\rangle$. The calculation of Fig. 1b is straightforward, without expansion in terms of the transverse momenta of the quarks and gluon. Furthermore, all these calculations have to be combined into the gauge invariant matrix elements, such as $G_{D}, \tilde{G}_{D}$, $H_{D}, E_{D}, T_{F}, \tilde{T}_{F}, T_{F}^{(\sigma)}, \tilde{T}_{F}^{(\sigma)}, \tilde{g}$, and $\tilde{h}$, which are constructed from the gauge invariant operators $\bar{\psi} D_{\perp} \psi$ and $\bar{\psi} F^{+\perp} \psi^{23}$.

With the large transverse momentum quark distributions calculated following the above procedure, one will be able to show that the differential cross section in the TMD factorization approach will be the same as the collinear factorization approach in the intermediate transverse momentum region. This consistency has been shown for the the SSA contributions coming from the polarized distributions of the incoming nucleon ${ }^{19,20}$. For the contribution from the twist-three fragmentation function, it has been difficult to show this consistency. However, recent studies on the universality property for the Collins fragmentation function in various processes have paved way to demonstrate this consistency between the TMD and collinear factorization approaches. This was finished in a recent publication ${ }^{24}$. In particular, the TMD quark fragmentation function are defined through the following matrix,

$$
\begin{aligned}
\mathcal{M}_{h}^{\alpha \beta}\left(z, p_{\perp}\right)= & \frac{n^{+}}{z} \int \frac{d \xi^{-}}{2 \pi} \frac{d^{2} \xi_{\perp}}{(2 \pi)^{2}} e^{-i\left(k^{+} \xi^{-}-\vec{k}_{\perp} \cdot \vec{\xi}_{\perp}\right)} \sum_{X} \frac{1}{3} \sum_{a}\left\langle 0\left|\mathcal{L}_{0} \psi_{\beta a}(0)\right| P_{h} X\right\rangle \\
& \times\left\langle P_{h} X\right|\left(\bar{\psi}_{\alpha a}\left(\xi^{-}, \vec{\xi}_{\perp}\right) \mathcal{L}_{\xi}^{\dagger}|0\rangle\right.
\end{aligned}
$$

where $a=1,2,3$ is a color index, $\alpha$ and $\beta$ are Dirac indices, and $p_{\perp}$ is the transverse momentum of the final state hadron with momentum $P_{h}$ relative to the fragmenting quark $k$. The quark momentum $k$ is dominated by its plus component $k^{+}=\left(k^{0}+\right.$ $\left.k^{z}\right) / \sqrt{2}$, and we have $P_{h}^{+}=z k^{+}$and $\vec{k}_{\perp}=-\vec{p}_{\perp} / z$. For convenience, we have chosen a vector $n=\left(1^{+}, 0^{-}, 0_{\perp}\right)$ which is along the plus momentum direction. The leading order expansion of the above matrix leads to two fragmentation functions for a 
scalar meson,

$$
\mathcal{M}_{h}=\frac{1}{2}\left[D\left(z, p_{\perp}\right) \not h+\frac{1}{M} H_{1}^{\perp}\left(z, p_{\perp}\right) \sigma^{\mu \nu} p_{\mu \perp} n_{\nu}\right]
$$

where $M$ is a mass scale chosen for convenience, and the second term defines the Collins function $H_{1}^{\perp}$. From the above equation, we can further define the transversemomentum moment of the Collins function: $\hat{H}(z)=\int d^{2} p_{\perp} \frac{p_{\perp}^{2}}{2 M} H_{1}^{\perp}\left(z, p_{\perp}\right)$. By integrating out the transverse momentum, the fragmentation function will only depend on the longitudinal momentum fraction $z$ of the quark carried by the final state hadron. It is straightforward to show that this function can be written as a twistthree matrix element of the fragmentation function,

$$
\begin{aligned}
\hat{H}(z)=n^{+} & z^{2} \int \frac{d \xi^{-}}{2 \pi} e^{i k^{+}} \xi^{-} \frac{1}{2}\left\{\operatorname{Tr} \sigma^{\alpha+}\left\langle 0\left|\left[i D_{\perp}^{\alpha}+\int_{\xi^{-}}^{+\infty} d \zeta^{-} g F^{\alpha+}\left(\zeta^{-}\right)\right] \psi(\xi)\right| P_{h} X\right\rangle\right. \\
& \left.\times\left\langle P_{h} X|\bar{\psi}(0)| 0\right\rangle+h . c .\right\}
\end{aligned}
$$

where $F^{\mu \nu}$ is the gluon field strength tensor and we have suppressed the gauge links between different fields and other indices for simplicity. From the above definition, we can see that $\hat{H}(z)$ involves derivative on the quark field and the field strength tensor explicitly, and it belongs to more general twist-three fragmentation functions. For example, extending the above definition, we can define a two-variable dependent twist-three fragmentation function as,

$$
\begin{aligned}
\hat{H}_{D}\left(z_{1}, z_{2}\right)= & n^{+} z_{1} z_{2} \int \frac{d \xi^{-} d \zeta^{-}}{(2 \pi)^{2}} e^{i k_{2}^{+} \xi^{-}} e^{i k_{g}^{+} \zeta^{-}} \frac{1}{2}\left\{\operatorname{Tr} \sigma^{\alpha+}\left\langle 0\left|i D_{\perp}^{\alpha}\left(\zeta^{-}\right) \psi\left(\xi^{-}\right)\right| P_{h} X\right\rangle\right. \\
& \left.\times\left\langle P_{h} X|\bar{\psi}(0)| 0\right\rangle+h . c .\right\},
\end{aligned}
$$

where $k_{i}^{+}=P^{+} / z_{i}$ and $k_{g}^{+}=k_{1}^{+}-k_{2}^{+}$. These functions are our starting point to formulate the Collins mechanism in the collinear factorization approach. First, we can calculate the transverse momentum dependence of the Collins function in the perturbative region from the twist-three fragmentation functions $\hat{H}_{D}$ and $\hat{H}$. To do this, we will have to not only calculate the perturbative diagrams with gluon radiation, but also to perform the twist expansion and take into account full contributions from the $\partial_{\perp}$ and $A_{\perp}$ operators in the definitions of $\hat{H}_{D}$ and $\hat{H}$ at this order. An important check of the above result is its universality property. Indeed, we find that our calculations are independent of the gauge link direction used in Eq. (6). Because of this, this calculation shall apply to all the processes the Collins function involved. This clearly demonstrates its universality property. Furthermore, we can also calculate the Collins contribution to the SSA in semi-inclusive DIS, $e p_{\uparrow} \rightarrow e^{\prime} \pi X$, and show that the TMD and collinear factorization approaches are consistent in the intermediate transverse momentum region $\Lambda_{\mathrm{QCD}} \ll P_{h \perp} \ll Q$, This clearly demonstrates that in the intermediate transverse momentum region, the twist-three collinear factorization approach and the TMD factorization approach provide a unique picture for the Collins contribution to the SSA in the semi-inclusive DIS ${ }^{24}$. 


\section{QCD Evolution and NLO Calculation for SSA Observable}

Most recently, there has been very exciting progress in studying the scale evolution equations for the quark-gluon and three-gluon correlation functions and their implications to the energy dependence of the relevant SSA observables ${ }^{26,27,28,29}$. General structure of the evolution equations for the twist-three quark-gluon correlation functions has been known in the literature ${ }^{30}$. In a very recent publication ${ }^{29}$, some interesting results are found (see the discussions below) in applying the known results from Ref. ${ }^{30}$ to the evolution equations derived in Refs. ${ }^{26,27,28}$. On the other hand, from the large transverse momentum quark Sivers function calculated in ${ }^{19}$, we would already obtain the evolution equation for $T_{F}(x)$ (which is the transverse momentum moment of the quark Sivers function), since the collinear divergence in that calculation will lead to the splitting function of $T_{F}(x)$. This splitting function was confirmed by a complete calculation of next-to-leading order QCD correction to the transverse-momentum weighted spin asymmetry in Drell-Yan lepton pair production ${ }^{26}$ and the derivations of the scale evolution equations directly ${ }^{27,28}$. In particular, the scale evolution for the quark-gluon correlation function $T_{F}(x)$ is found to be (quark-quark splitting kernel),

$$
\begin{aligned}
\frac{\partial}{\partial \ln \mu^{2}} T_{F}\left(x_{B}, \mu^{2}\right)= & \frac{\alpha_{s}}{2 \pi} \int \frac{d x}{x}\left[C_{F}\left\{\frac{1+z^{2}}{(1-z)_{+}}+\frac{3}{2} \delta(1-z)\right\} T_{F}(x, x)\right. \\
& \left.+\frac{C_{A}}{2}\left\{\frac{1+z}{1-z} T_{F}(x z, x)-\frac{1+z^{2}}{1-z} T_{F}(x, x)+\tilde{T}_{F}(x z, x)\right\}\right]
\end{aligned}
$$

where $z=x_{B} / x$. In Ref. ${ }^{29}$, it has been found two additional contributions for this splitting contribution. One term comes from the so-called New-Hard-Pole contribution ${ }^{31}$. After taking this contribution, the calculations in ${ }^{26}$ will be able to reproduce this term. Another terms is associated with a Delta function in the splitting kernel and a color-factor $C_{A}$, which has not yet been identified in the calculation approach in Refs. ${ }^{26,27,28}$. It is highly important to have an independent calculation to check this term from the general evolution equations for the twist-three operators.

Furthermore, the NLO perturbative-QCD correction to the transverse momentum weighted single spin asymmetry in Drell-Yan lepton pair production in hadronic collisions has engaged the transverse spin physics to a more solid theoretical ground ${ }^{26}$. It has been shown that the collinear divergences can be absorbed into the NLO twist-three quark-gluon correlation function of the transversely polarized nucleon and the unpolarized quark distribution of the unpolarized nucleon. This calculation suggests that a general factorization formula exists for the transverse momentum weighted spin-dependent cross section in the Drell-Yan process, in extension of the general factorization arguments given in ${ }^{32}$.

One important feature of this result is its behavior near "partonic threshold", that is in the large- $z$ limit of the integrand, corresponding to $\hat{s} \sim Q^{2}$, when the initial partons have "just enough" energy to produce the virtual photon. Setting 
the scale $\mu=Q$, we have the following structure of the NLO correction in this case:

$$
\begin{aligned}
\frac{d\left\langle q_{\perp} \Delta \sigma\left(S_{\perp}\right)\right\rangle}{d Q^{2}}=\sigma_{0} & \frac{\alpha_{s}}{2 \pi} \int \frac{d x}{x} \frac{d x^{\prime}}{x^{\prime}} T_{F}\left(x, x ; \mu^{2}\right) \bar{q}\left(x^{\prime} ; \mu^{2}\right) \\
& \times\left[4 C_{F}\left(1+z^{2}\right)\left(\frac{\ln (1-z)}{1-z}\right)_{+}\right],
\end{aligned}
$$

where we only keep the "double-logarithmic" term which dominates near threshold in the $\overline{\mathrm{MS}}$ scheme. The structure of this expression is identical to that for the spinaveraged $q_{\perp}$-integrated NLO cross section near threshold,

$$
\frac{d \sigma}{d Q^{2}}=\sigma_{0} \frac{\alpha_{s}}{2 \pi} \int \frac{d x}{x} \frac{d x^{\prime}}{x^{\prime}} q\left(x ; \mu^{2}\right) \bar{q}\left(x^{\prime} ; \mu^{2}\right)\left[4 C_{F}\left(1+z^{2}\right)\left(\frac{\ln (1-z)}{1-z}\right)_{+}\right] \text {. }
$$

This means that the soft gluon contribution is spin-independent. It contributes in the same way to the spin-averaged and single-spin-dependent cross sections, and will lead to the same soft-gluon threshold resummation effects to these cross sections, at least at the leading double logarithmic level. This observation is very similar to that made for the transverse momentum resummation in the Drell-Yan process ${ }^{33}$. This will likely have the phenomenological consequence that the single-spin asymmetry for the Drell-Yan process will be quite stable under NLO corrections, in particular when $\tau=Q^{2} / s$ is large.

In summary, there have been great progresses in transverse spin physics in the last few years. Unfortunately, I could not cover all these important developments, and rather I emphasized a few examples, including the universality of the parton distribution and fragmentation functions, and QCD evolution and next-to-leading order corrections to the relevant observables. On the other hand, there are many excellent talks on transverse spin physics in this conference, and I believe that they

will present more comprehensive reviews on these exciting developments in this physics.

\section{Acknowledgments}

This work was supported in part by the U.S. Department of Energy under contract DE-AC02-05CH11231. We are grateful to RIKEN, Brookhaven National Laboratory and the U.S. Department of Energy (contract number DE-AC02-98CH10886) for providing the facilities essential for the completion of this work.

\section{References}

1. A. V. Efremov and O. V. Teryaev, Sov. J. Nucl. Phys. 36, 140 (1982) [Yad. Fiz. 36, 242 (1982)]; A. V. Efremov and O. V. Teryaev, Phys. Lett. B 150, 383 (1985).

2. J. Qiu and G. Sterman, Phys. Rev. Lett. 67, 2264 (1991); Nucl. Phys. B 378, 52 (1992); Phys. Rev. D 59, 014004 (1999).

3. G. L. Kane, J. Pumplin and W. Repko, Phys. Rev. Lett. 41, 1689 (1978). 
4. see the contributions by M. Anselmino, S. Boffi, F. Bradamate, F. Ceccopieri, L. Gamberg, A. Metz, B. Pasquini, P. Ratcliffe, P. Schweitzer, N. Stefanis, T. Teckentrup, O. Teryaev, these proceedings.

5. D. W. Sivers, Phys. Rev. D 41, 83 (1990); Phys. Rev. D 43, 261 (1991).

6. J. C. Collins, Nucl. Phys. B 396, 161 (1993).

7. S. J. Brodsky, D. S. Hwang and I. Schmidt, Phys. Lett. B 530, 99 (2002); Nucl. Phys. B 642, 344 (2002).

8. J. C. Collins, Phys. Lett. B 536, 43 (2002).

9. X. Ji and F. Yuan, Phys. Lett. B 543, 66 (2002); A. V. Belitsky, X. Ji and F. Yuan, Nucl. Phys. B 656, 165 (2003).

10. D. Boer, P. J. Mulders and F. Pijlman, Nucl. Phys. B 667, 201 (2003).

11. A. Metz, Phys. Lett. B 549, 139 (2002).

12. J. C. Collins and A. Metz, Phys. Rev. Lett. 93, 252001 (2004).

13. L. P. Gamberg, A. Mukherjee and P. J. Mulders, Phys. Rev. D 77, 114026 (2008).

14. F. Yuan, Phys. Rev. Lett. 100, 032003 (2008); Phys. Rev. D 77, 074019 (2008).

15. S. Meissner and A. Metz, Phys. Rev. Lett. 102, 172003 (2009).

16. C. J. Bomhof, P. J. Mulders and F. Pijlman, Phys. Lett. B 596, 277 (2004); Eur. Phys. J. C 47, 147 (2006); JHEP 0702, 029 (2007); A. Bacchetta, C. J. Bomhof, P. J. Mulders and F. Pijlman, Phys. Rev. D 72, 034030 (2005); C. J. Bomhof and P. J. Mulders, arXiv:0709.1390 [hep-ph].

17. J. W. Qiu, W. Vogelsang and F. Yuan, Phys. Lett. B 650, 373 (2007); Phys. Rev. D 76, 074029 (2007); W. Vogelsang and F. Yuan, Phys. Rev. D 76, 094013 (2007).

18. J. Collins and J. W. Qiu, Phys. Rev. D 75, 114014 (2007); J. Collins, arXiv:0708.4410 [hep-ph].

19. X. Ji, J. W. Qiu, W. Vogelsang and F. Yuan, Phys. Rev. Lett. 97, 082002 (2006); Phys. Rev. D 73, 094017 (2006); Phys. Lett. B 638, 178 (2006).

20. Y. Koike, W. Vogelsang and F. Yuan, Phys. Lett. B 659, 878 (2008).

21. J. Zhou, F. Yuan and Z. T. Liang, Phys. Rev. D 78, 114008 (2008).

22. J. Zhou, F. Yuan and Z. T. Liang, Phys. Lett. B 678, 264 (2009).

23. J. Zhou, F. Yuan and Z. T. Liang, arXiv:0909.2238 [hep-ph].

24. F. Yuan and J. Zhou, Phys. Rev. Lett. 103, 052001 (2009).

25. X. Ji, J. P. Ma and F. Yuan, Phys. Rev. D 71, 034005 (2005); Phys. Lett. B 597, 299 (2004).

26. W. Vogelsang and F. Yuan, Phys. Rev. D 79, 094010 (2009).

27. Z. B. Kang and J. W. Qiu, Phys. Rev. D 79, 016003 (2009).

28. J. Zhou, F. Yuan and Z. T. Liang, Phys. Rev. D 79, 114022 (2009).

29. V. M. Braun, A. N. Manashov and B. Pirnay, arXiv:0909.3410 [hep-ph].

30. See, for example: I. I. Balitsky and V. M. Braun, Nucl. Phys. B 311, 541 (1989); P. G. Ratcliffe, Nucl. Phys. B 264, 493 (1986); X. D. Ji and C. h. Chou, Phys. Rev. D 42, 3637 (1990); A. V. Belitsky and D. Mueller, Nucl. Phys. B 503, 279 (1997); I. I. Balitsky, V. M. Braun, Y. Koike and K. Tanaka, Phys. Rev. Lett. 77, 3078 (1996).

31. Y. Koike and K. Tanaka, arXiv:0907.2797 [hep-ph].

32. J. W. Qiu and G. Sterman, Nucl. Phys. B 353, 105 (1991); Nucl. Phys. B 353, 137 (1991).

33. A. Idilbi, X. d. Ji, J. P. Ma and F. Yuan, Phys. Rev. D 70, 074021 (2004). 